Pacific Journal of Mathematics

RR A OF LINKED OPERATORS 


\title{
ON THE SPECTRA OF LINKED OPERATORS
}

\author{
Charles J. A. Halberg Jr. and Angus E. Taylor
}

1. Introduction. Let $X, Y$ be complex linear spaces, and $Z$ a non-void complex linear space contained in both $X$ and $Y$. Let $X$ be a Banach space $X_{1}, Y$ a Banach space $Y_{2}$ under the norms $n_{1}, n_{2}$ respectively. Let $Z$ be a Banach space $Z_{N}$ under the norm $N$ defined by $N(z)=\max \left[n_{1}(z), n_{2}(z)\right]$. (This is equivalent to saying that if $\left\{z_{n}\right\}$ is any sequence with $z_{n} \in Z$, such that $z_{n} \rightarrow x$ in the topology of $X_{1}$ and $z_{n} \rightarrow y$ in the topology of $Y_{2}$, then $x=y \in Z$. Our particular method of stating this here will be useful for later purposes.) With the usual uniform norms let $T_{1}, T_{2}$ be bounded distributive operators on $X_{1}, Y_{2}$ respectively, such that $T_{1} z=T_{2} z \in Z$ when $z \in Z$. Operators satisfying these conditions will be said to be "linked". If, in addition, it is assumed that $Z$ is dense in $X_{1}, T_{1}$ and $T_{2}$ will be said to be "linked densely relative to $X_{1}^{\prime \prime}$.

We are interested in relationships between the spectra of linked operators. That there are linked, and densely linked operators with different spectra will be shown in $\S 3$. The main result of this paper is the demonstration that, if $T_{1}$ and $T_{2}$ are linked densely relative to $X_{1}$, under certain circumstances any component of the spectrum of $T_{1}$ has a non-void intersection with the spectrum of $T_{2}$. Sufficient conditions are that if $\lambda$ belongs to the intersection of the resolvent sets of $T_{1}$ and $T_{2}$ and $z \in Z$, then $\left(\lambda I-T_{1}\right)^{-1} z=\left(\lambda I-T_{2}\right)^{-1} z \in Z$. With this result we obtain some interesting consequences in the special case where the Banach spaces considered are the sequence spaces $l_{p}$.

2. Preliminary definitions and notation. Supposing $X$ to be a complex linear space such that under a norm $n_{a},\left(x \in X, n_{a}(x)=\|x\|_{a}\right), X$ becomes a complex Banach space $X_{a}$, we let $\left[X_{a}\right]$ denote the set of all operators $T$ that are bounded under the induced norm

$$
\|T\|_{a}=\sup \|T x\|_{a} \text { (for all } x \in X_{a},\|x\|_{a}=1 \text { ). }
$$

Such a $T$ will be denoted by $T_{a}$ when considered as an element of the algebra $\left[X_{a}\right]$. If $T_{a} \in\left[X_{a}\right]$ we classify all complex numbers into two sets :

(1) The resolvent set $\rho\left(T_{a}\right)$, consisting of all $\lambda$ such that $\lambda I-T_{a}$ defines a one-to-one correspondence of $X_{a}$ onto $X_{a}$.

(2) The spectrum $\sigma\left(T_{a}\right)$, consisting of all $\lambda$ not in $\rho\left(T_{a}\right)$.

The spectrum is divided into three parts:

Received February 1, 1955. 
(1) The point spectrum $p\left(T_{a}\right)$, consisting of those $\lambda$ for which $\left(\lambda I-T_{a}\right)^{-1}$ does not exist.

(2) The continuous spectrum $c\left(T_{a}\right)$, consisting of those $\lambda$ not in $\rho\left(T_{a}\right)$ or $p\left(T_{a}\right)$ for which the range of $\lambda I-T_{a}$ is dense in $X_{a}$; and

(3) The residual spectrum $r\left(T_{a}\right)$, consisting of those $\lambda$ not in $\rho\left(T_{a}\right)$. $p\left(T_{a}\right)$ or $c\left(T_{a}\right)$.

We shall also have occasion to refer to the so-called "approximate point spectrum," consisting of those $\lambda$ for which $\left(\lambda I-T_{a}\right)^{-1}$ is not bounded. It is well known that $\sigma\left(T_{a}\right)$ is closed, bounded and nonempty. It is also well known that $R_{\lambda}\left(T_{a}\right) \equiv\left(\lambda I-T_{a}\right)^{-1}$ is analytic in $\rho\left(T_{a}\right)$ as a function with values in $\left[X_{a}\right]$.

3. An example of linked operators with different spectra. Consider the well known sequence spaces $l_{1}$ and $l_{2}$. Let $T_{1}$ and $T_{2}$ be the operation defined as elements of $\left[l_{1}\right]$ and $\left[l_{2}\right]$ respectively by the infinite matrix $\left(t_{i j}\right)$

$$
t_{i j}=\left\{\begin{array}{clc}
\frac{j}{(i-1) i} & \text { if } & i>j \\
0 & \text { if } & i \leqq j
\end{array}\right.
$$

The uniform norm for the operator $T$ defined by such a matrix, when considered as an operator on $l_{1}$, can be shown to be the supremum of the $l_{1}$ norms of the column sequences of the matrix $\left(t_{i j}\right)$ :

$$
\left\|T_{1}\right\|_{1}=\sup _{j} \sum_{i=1}^{\infty}\left|t_{i j}\right|
$$

([1, pp. 696-697]). From this it is easy to see that $\left\|T_{1}\right\|_{1}=1$. In fact

$$
\sum_{i=1}^{\infty}\left|t_{i j}\right|=j \sum_{i=j+1}^{\infty} \frac{1}{(i-1) i}=j \frac{1}{j}=1,
$$

the sum being independent of $j$. Next, considering the powers of $T$ :

$$
T^{n}=\left(t_{i j}\right)^{n}=\left(t_{i j}^{(n)}\right),
$$

we see that

$$
\left\|T_{1}^{2}\right\|_{1} \equiv \sup _{j} \sum_{i=1}^{\infty}\left|t_{i j}^{(2)}\right|=\sup _{j} \sum_{i=1}^{\infty}\left|\sum_{k=1}^{\infty} t_{i k} t_{k j}\right|=\sup _{j} \sum_{k=1}^{\infty}\left(\sum_{i=1}^{\infty} t_{i k}\right) t_{k j}=\sup _{j} \sum_{k=1}^{\infty} t_{k j}=1 .
$$

By induction it is easy to show that $\sum_{i=1}^{\infty}\left|t_{i j}^{(n)}\right|=1$ for any $j$, and hence $\left\|T_{1}^{n}\right\|_{1}=1$. Now it is well known that the spectral radius of $T_{1}$,

$$
\left|\sigma\left(T_{1}\right)\right| \equiv \sup |\lambda|, \quad\left(\text { for } \lambda \in o\left(T_{1}\right)\right) \text {, }
$$


is given by the formula

$$
\left|\sigma\left(T_{1}\right)\right|=\lim _{n \rightarrow \infty}\left(\left\|T_{1}^{n}\right\|_{1}\right)^{1 / n} ;
$$

hence the spectral radius of $T_{1}^{\prime}$ is 1 .

On the other hand, by making use of an inequality due to Schur $\left[2\right.$, p. 6], we can estimate the norm of $T$ as an operator on $l_{2}$ :

$$
\left\|T_{2}\right\|_{2} \leqq\left[\left(\sup _{i} \sum_{j=1}^{\infty}\left|t_{i j}\right|\right)\left(\sup _{j} \sum_{i=1}^{\infty}\left|t_{i j}\right|\right)\right]^{\frac{1}{2}} .
$$

In this way we see that

$$
\left\|T_{2}\right\|_{2} \subseteq V 1 \cdot \frac{1}{2}<1
$$

since

$$
\sum_{j=1}^{\infty}\left|t_{i j}\right|=\sum_{j=1}^{i-1} \frac{j}{(i-1) i}=\frac{1}{2}
$$

the sum being independent of $i$. Since it is always true that $\left|\sigma\left(T_{2}\right)\right| \leqq\left\|T_{2}\right\|_{2}$, it is now clear that $\left|\sigma\left(T_{2}\right)\right|<\left|\sigma\left(T_{1}\right)\right|$, whence we immediately infer that there exists a $\lambda$ such that $\lambda \in \sigma\left(T_{1}\right)$ and $\lambda \notin \sigma\left(T_{2}\right)$.

4. The projection corresponding to a spectral set. For the proof of our main theorem we need the concepts of spectral set and the projection associated with a spectral set. For this purpose we introduce the following definitions.

Suppose $X$ is a complex Banach space, and $T$ an element of $[X]$. A set $\sigma$ in the complex plane is called a spectral set of $T$ if $\sigma \subset \sigma(T)$ and if $\sigma$ is both open and closed in the relative topology of $\sigma(T)$.

If $\sigma$ is a spectral set of $T$, the corresponding projection is the operator defined by

$$
E_{o}[T]=\frac{1}{2 \pi i} \int R_{\lambda}(T) d \lambda,
$$

the integral being extended in the positive sense around the boundary of a suitable bounded open set $D$ such that $\sigma \subset D$ and the closure of $D$ does not intersect the rest of $\sigma(T)$. It is easy to see that if $\Delta$ is a closed set which does not intersect $\sigma$, the set $D$ may be chosen to satisfy the additional requirement that its closure does not intersect $\Delta$.

We now proceed to the proof of our main theorem.

5. Relations between the spectra of linked operators. Let $X$ and $Y$ be complex linear spaces such that $X$ becomes a Banach space $X_{1}$ and $Y$ becomes a Banach space $Y_{2}$ under the norms $n_{1}$ and $n_{2}$, respectively. 
THEOREM. Let $T_{1} \in\left[X_{1}\right]$ and $T_{2} \in\left[Y_{2}\right]$ be linked densely relative to $X_{1}$ and let $Z \subset X \cap Y$ be a complex linear space that becomes a Banach space $Z_{N}$ under the norm $N$ defined by $N(z)=\max \left[n_{1}(z), n_{2}(z)\right]$. Let $R_{\lambda}\left(T_{1}\right) z=$ $R_{\lambda}\left(T_{2}\right) z \in Z$ for every $z \in Z$, provided that $\lambda \in \rho\left(T_{1}\right) \cap \rho\left(T_{2}\right)$. Then if $C$ is any component of $\sigma\left(T_{1}\right), C \cap \sigma\left(T_{2}\right)$ is non-void.

Proof. We shall first prove that if $\sigma$ is any non-void spectral set of $\sigma\left(T_{1}\right)$, then $\sigma \bigcap \sigma\left(T_{2}\right)$ is non-void.

Suppose that $\sigma \cap \sigma\left(T_{2}\right)$ is void. Let $E_{\sigma}\left[T_{1}\right]$ be the projection in $\left[X_{1}\right]$ associated with $\sigma$, that is

$$
E_{\sigma}\left[T_{1}\right]=\frac{1}{2 \pi i} \int_{+B(D)} R_{\lambda}\left(T_{1}\right) d \lambda,
$$

where $B(D)$ is the boundary of a bounded Cauchy domain such that $\sigma \subset D$ while the closure of $D$ intersects neither $\sigma\left(T_{2}\right)$ nor the rest of $\sigma\left(T_{1}\right)$. We know that $E_{\sigma}\left[T_{1}\right] \neq 0$ by a theorem [3, p. 210] which states that the spectral set $\sigma$ is empty if and only if $E_{\sigma}\left[T_{1}\right]=0$. Now consider the operator (an element of $\left[Y_{2}\right]$ )

$$
F \equiv \frac{1}{2 \pi i} \int_{+B(D)} R_{\lambda}\left(T_{2}\right) d \lambda
$$

Since $D$ and $B(D)$ lie in $\rho\left(T_{2}\right), R_{\lambda}\left(T_{2}\right)$ is analytic inside and on $B(D)$; therefore the integral defining $F$ is the zero element of $\left[Y_{2}\right]$, by Cauchy's theorem.

If $\lambda \in \rho\left(T_{1}\right) \cap \rho\left(T_{2}\right)$, then by hypothesis $R_{\lambda}\left(T_{1}\right) z=R_{\lambda}\left(T_{2}\right) z$ for $z \in Z$, and from this we see that

$$
F z=E_{\sigma}\left[T_{1}\right] z \text { for } z \in Z \text {, }
$$

since the integrals defining $F z$ and $E_{\sigma}\left[T_{1}\right] z$ can be regarded as limits, in $Y_{2}$ and $X_{1}$ respectively, of the same sequence in $Z$. However, since $E_{o}\left[T_{1}\right] \neq 0$ and is continuous, and $Z$ is dense in $X_{1}$, there exists a $z$, $z \in Z$, such that $E_{\sigma}\left[T_{1}\right] z \neq 0$. But $F z=0$, which is a contradiction. Thus any non-void spectral set of $\sigma\left(T_{1}\right)$ has a non-void intersection with $\sigma\left(T_{2}\right)$.

Let $C$ be any component of $\sigma\left(T_{1}\right)$. To show that $C \cap \sigma\left(T_{2}\right)$ is nonvoid we will need the following theorem [4, p. 15]: If $A$ and $B$ are disjoint closed subsets of a compact set $K$ such that no component of $K$ intersects both $A$ and $B$, there exists a separation $K=K_{1} \cup K_{2}$, where $K_{1}$ and $K_{2}$ are disjoint compact sets containing $A$ and $B$ respectively. Now suppose that $C \cap\left(\sigma\left(T_{1}\right) \cap \sigma\left(T_{2}\right)\right)$ is void. Then, since $C$ and $\sigma\left(T_{1}\right) \cap \sigma\left(T_{2}\right)$ are non-void disjoint closed subsets in $\sigma\left(T_{1}\right)$ and as the only component of $\sigma\left(T_{1}\right)$ intersecting $C$ is $C$ itself, we have $\sigma\left(T_{1}\right)=K_{1} \bigcup K_{2}$, where $K_{1} \supset C, K_{2} \supset \sigma\left(T_{1}\right) \cap \sigma\left(T_{2}\right)$, and $K_{1}, K_{2}$ are disjoint compact sets. But $K_{1}$ is closed, being compact, and also relatively open, since it is the rela- 
tive complement of the closed set $K_{2}$. Thus $K_{1}$ is a spectral set of $\sigma\left(T_{1}\right)$, and $K_{1} \cap\left(\sigma\left(T_{1}\right) \cap \sigma\left(T_{2}\right)\right)$ is void, which is in contradiction to what we have shown above. Thus if $C$ is any component of $\sigma\left(T_{1}\right)$, then $C \cap \sigma\left(T_{2}\right)$ is non-void, as was to be proved.

We note that if in the hypotheses of the theorem we only require $T_{2}$ to be a closed distributive operator on $Y_{2}$, such that $\sigma\left(T_{2}\right)$ is nonvoid, the conclusion and proof of the theorem will be unaltered. Also, if we replace the hypotheses that $T_{1} \in\left[X_{1}\right]$ and $T_{2} \in\left[Y_{2}\right]$ by " $T_{1}$ and $T_{2}$ are closed distributive operators on $X_{1}$ and $Y_{2}$ respectively, such that $\sigma\left(T_{2}\right)$ is nonvoid", and retain the remaining hypotheses, we can conclude, using the same reasoning as before, that any non-void bounded spectral set of $\sigma\left(T_{1}\right)$ has a non-void intersection with $\sigma\left(T_{2}\right)$.

A very special case of our theorem, but one of considerable practical importance, is given in the following corollary.

COROLlaRY 1. In addition to the hypotheses of the preceding theorem let $Z$ be dense in $Y_{2}$, and let $\sigma\left(T_{1}\right)$ and $\sigma\left(T_{2}\right)$ be such that all of their components are single points. Then $\sigma\left(T_{1}\right)=\sigma\left(T_{2}\right)$.

In the special case where $X \subset Y$, the operators $T_{1} \in\left[X_{1}\right], T_{2} \in\left[Y_{2}\right]$ are linked, and $X$ plays the role of $Z$, we have the following two corollaries.

Corollary 2. If $C$ is any component of $\sigma\left(T_{1}\right)$, then $C \cap \sigma\left(T_{2}\right)$ is non-void.

Proof. This follows from the theorem, since if $\lambda \in \rho\left(T_{1}\right) \cap \rho\left(T_{2}\right)$, then $R_{\lambda}\left(T_{1}\right) x=R_{\lambda}\left(T_{2}\right) x$ for $x \in X$.

Corolllary 3. If $T_{1}$ and $T_{2}$ are linked densely relative to $Y_{2}$ and $C$ is any component of $\sigma\left(T_{2}\right)$, then $C \cap \sigma\left(T_{1}\right)$ is non-void.

This should be clear from the proof of the theorem in view of the remark following the statement of Corollary 2.

Definition. If $A, B, C$ are sets such that any component of $C$ has a non-void intersection with both $A$ and $B$ we shall say that $A$ and $B$ are "linked by $C$ ". If in addition every component of $A$ has a non-void intersection with $C$ we shall say that $A$ is "totally linked to $B$ by $C$ ".

Now suppose that neither $X$ nor $Y$ is necessarily contained in the other and let $T \in\left[Z_{N}\right]$ be the operator defined by $T z=T_{1} z$ for $z \in Z$. Then we have the following results for $T_{1} \in\left[X_{1}\right]$ and $T_{2} \in\left[Y_{2}\right]$. 
CoRollary 4. If $T_{1}$ and $T_{2}$ are linked (not necessarily densely linked), then $\sigma\left(T_{1}\right)$ and $\sigma\left(T_{2}\right)$ are linked by $\sigma(T)$.

This follows immediately from Corollary 2.

Corollary 5. If $T_{1}$ and $T_{2}$ are linked densely relative to $X_{1}$, then $\sigma\left(T_{1}\right)$ is totally linked to $\sigma\left(T_{2}\right)$ by $\sigma(T)$.

This follows from Corollary 3.

CoRollaRy 6. If $T_{1}$ and $T_{2}$ are linked, then

$$
\sigma(T)-\left(\sigma\left(T_{1}\right) \cup \sigma\left(T_{2}\right)\right)
$$

is contained in that portion of the residual spectrum of $T$ for which $(\lambda I-T)^{-1}$ is bounded.

Proof. Clearly $p(T)$ is contained in both $p\left(T_{1}\right)$ and $p\left(T_{2}\right)$. If $\lambda$ belongs to the approximate point spectrum of $T$ then there exists a sequence $\left\{z_{n}\right\}, z_{n} \in Z$, such that

$$
\lim _{n \rightarrow \infty}\left\|(\lambda I-T) z_{n}\right\|_{N}=0 \text { and }\left\|z_{n}\right\|_{N}=1 .
$$

But either $1^{\circ}$ : ' Infinitely many $z_{n}$ are such that $\left\|z_{n}\right\|_{n_{1}}=1$, or $2^{\circ}$ : Infinitely many $z_{n}$ are such that $\left\|z_{n}\right\|_{n_{2}}=1$. If $1^{\circ}$ holds there exists a subsequence $\left\{x_{n}\right\}$ of $\left\{z_{n}\right\}$ such that

$$
\lim _{n \rightarrow \infty}\left\|(\lambda I-T) x_{n}\right\|_{n_{1}}=0 \text { and }\left\|x_{n}\right\|_{n_{1}}=1,
$$

and thus $\lambda$ belongs to the approximate point spectrum of $T_{1}$. If $2^{\circ}$ holds similar reasoning shows that $\lambda$ belongs to the approximate point spectrum of $T_{2}$. From these results it follows that the only possibility for an element $\lambda, \lambda \in \sigma(T)$, to be such that $\lambda \notin \sigma\left(T_{1}\right) \cup \sigma\left(T_{2}\right)$ is for $\lambda$ to be an element of the residual spectrum of $T$ with $(\lambda I-T)^{-1}$ bounded.

The following is a corollary concerning the sequence spaces $l_{n}$, which we considered earlier.

COROLlaRY 7. Suppose that $1 \leqq r<s$, and suppose that the infinite matrix $\left(t_{i j}\right)$ defines operators $T_{r}$ and $T_{s}$ on $l_{r}$ and $l_{s}$, respectively, such that $T_{r} \in\left[l_{r}\right]$ and $T_{s} \in\left[l_{s}\right]$. Then $C \cap \sigma\left(T_{s}\right)$ is non-void for any component $C$ of $\sigma\left(T_{r}\right)$. Moreover, $C \cap \sigma\left(T_{r}\right)$ is non-void for any component $C$ of $\sigma\left(T_{s}\right)$.

Proof. These are special cases of Corollaries 2 and 3, for it is well known that, for the classes $l_{r}$ and $l_{s}$, we have $l_{r} \subset l_{s}$; that $\|x\|_{s} \leqq\|x\|_{r}$ for $x \in l_{r}$; and that $l_{r}$ is dense in $l_{s}$. 
Corollary 7 is true even if $s=\infty$. (We recall that $l_{\infty}$ is the set of all sequences $x=\left\{\xi_{i}\right\}$ such that $\sup \left|\xi_{i}\right|<\infty$, and such that if $x \in l_{\infty}$, $\left.\|x\|_{\infty}=\sup \left|\xi_{i}\right|.\right) \quad$ For, although in this case it is not true that $l_{r}$ is dense in $l_{\infty}$, the following is true: if an element of $\left[l_{\infty}\right]$ is defined by an infinite matrix, and if the operator is 0 when restricted to $l_{r}$, then it is the zero operator on $l_{\infty}$. The reasoning of the main theorem now applies with only slight modifications for the case in which $X_{1}=l_{\infty}$, $Y_{2}=l_{r}(1 \leqq r<\infty), Z=l_{r}$ and $T_{1}$ and $T_{2}$ are defined by the same matrix.

Before stating the final corollary we recall the following facts.

If $1<p \leqq \infty$ and $1 / p+1 / p^{\prime}=1$ (with $p^{\prime}=1$ if $p=\infty$ ), we can identify the conjugate space $\left(l_{p^{\prime}}\right)^{*}$ with $l_{p}$. If $\left(t_{i j}\right)$ is an infinite matrix defining a bounded linear operator $T$ on $l_{p^{\prime}}$, we can identify the adjoint operator $T^{*}$ with the bounded linear operator $T^{t}$ defined on $l_{p}$ by the transposed matrix $\left(t_{i j}^{t}\right)$, where $t_{i j}^{t}=t_{j i}$. Since $\sigma(T)=\sigma\left(T^{*}\right)$, as is well known [5, pp. 304 and 306], we have $\sigma\left(T_{p^{\prime}}\right)=\sigma\left(T_{p}^{t}\right)$, where the subscripts serve to remind us on what space the operator is defined.

COROLlaRY 8. Suppose the matrix $\left(t_{i j}\right)$ defines $T_{p} \in\left[l_{p}\right]$ and $T_{p^{\prime}} \in\left[l_{p^{\prime}}\right]$, where $1<p \leqq \infty$. Then $C \cap \sigma\left(T_{p}^{t}\right)$ is non-void for any component $C$ of $\sigma\left(T_{p}\right)$, and $C \cap \sigma\left(T_{p}\right)$ is non-void for any component $C$ of $\sigma\left(T_{p}^{t}\right)$.

Proof. This follows from Corollary 7 and the foregoing remarks, by taking $p$ and $p^{\prime}$ to be $r$ and $s$ or $s$ and $r$, depending on whether $p \leqq 2$ or $2<p$.

6. Further comments. The referee made some suggestions concerning the condition which was imposed in the main theorem of $\S 5$, namely that

$$
R_{\lambda}\left(T_{1}\right) z=R_{\lambda}\left(T_{2}\right) z \in Z \text { if } z \in Z \text { and } \lambda \in \rho\left(T_{1}\right) \bigcap \rho\left(T_{2}\right) .
$$

We shall refer to this as Condition (R). We add some discussion of this condition, guided in part by the suggestions of the referee.

As in $\S 5$, let us denote by $T$ the member of $\left[Z_{N}\right]$ defined by $T z=T_{1} z=T_{2} z$ when $z \in Z$. It is then easy to see that $R_{\lambda}(T) z=R_{\lambda}\left(T_{k}\right) z \in Z$ if $z \in Z$ and $\lambda \in \rho(T) \wedge \rho\left(\mathrm{T}_{k}\right), k=1,2$. Consequently $R_{\lambda}\left(T_{1}\right) z=R_{\lambda}\left(T_{2}\right) z \in Z$ if $z \in \rho(T) \cap \rho\left(T_{1}\right) \cap \rho\left(T_{2}\right)$. The intersection of these three resolvent sets certainly contains all sufficiently large values of $\lambda$. Now let $D$ be the set of those $\lambda \in \rho\left(T_{1}\right) \cap \rho\left(T_{2}\right)$ for which $R_{\lambda}\left(T_{1}\right) z=R_{\lambda}\left(T_{2}\right) z \in Z$ if $z \in Z$. This set is evidently closed relative to $\rho\left(T_{1}\right) \cap \rho\left(T_{2}\right)$ (by the continuity of the resolvents and the way in which the norm of $Z$ is defined). It is also open relative to $\rho\left(T_{1}\right) / \cap \rho\left(T_{2}\right)$, as we may see by using the expansion 


$$
R_{\lambda}=\sum_{n=j}^{\infty}(\mu-\lambda)^{n} R_{\mu}{ }^{n+1}
$$

for the resolvent of an operator in the neighborhood of a point $\mu$ in the resolvent set. Consequently $D$ contains all of any particular component of $\rho\left(T_{1}\right) \cap \rho\left(T_{2}\right)$ if it contains any point of that component. In particular $D$ contains all of the unbounded component of $\rho\left(T_{1}\right) \cap \rho\left(T_{2}\right)$. This shows that we can omit the Condition (R) if $\rho\left(T_{1}\right) \cap \rho\left(T_{2}\right)$ has only one component. In particular this will be true if $\sigma\left(T_{1}\right)$ and $\sigma\left(T_{2}\right)$ are totally disconnected. From what was said previously it is clear that $\rho\left(T_{1}\right) \cap \rho\left(T_{2}\right)-D$ lies in $\sigma(T)-\left(\sigma\left(T_{1}\right) \cup \sigma\left(T_{2}\right)\right)$, and hence, by Corollary 6, in that part of $\sigma(T)$ for which $(\lambda I-T)^{-1}$ exists and is bounded. It is not very difficult to prove that a point of this latter kind is not in the closure of $\rho(T)$. (The argument uses the functional equation of the resolvent, $R_{\lambda}-R_{\mu}=(\mu-\lambda) R_{\lambda} R_{\mu}$, to show that if $\alpha \in \overline{\rho(T)}$ then $\lim _{\lambda \rightarrow \infty} R_{\lambda}$ exists and is necessarily $R_{\alpha}$.) Consequently we see that $D$ contains the set $\overline{\rho(T)} \cap \rho\left(T_{1}\right) \cap \rho\left(T_{2}\right)$. This shows, for example, that Condition (R) is superfluous if $\rho(T)$ is everywhere dense in the plane.

\section{REFERENCES}

1. L. W. Cohen and Nelson Dunford, Transformations on sequence spaces, Duke Math. J., 3, (1937), 689-701.

2. J. Schur, Bemerkungen zur Theorie der beschränkten Bilinearformen mit unendlich vielen Veränderlichen, J. für Math., 140 (1911), 1-28.

3. A. E. Taylor, Spectral theory of closed distributive operators, Acta. Math., 84 (1950), 189-224.

4. G. T. Whyburn, Analytic topology, Amer. Math. Soc. Colloquium Publications, 28, New York, 1942.

5. A. C. Zaanen, Linear analysis, Interscience, New York, 1953. 


\section{PACIFIC JOURNAL OF MATHEMATICS}

EDITORS

\author{
H. L. Royden \\ Stanford University \\ Stanford, California \\ E. Hewite \\ University of Washington \\ Seattle 5 , Washington
}

\author{
R. P. Dilworth \\ California Institute of Technology \\ Pasadena 4, California
}

\author{
A. Horn* \\ University of California \\ Los Angeles 24, California
}

\section{ASSOCIATE EDITORS}
E. F. BECKENBACH
M. HALL
M. S. KNEBELMAN
J. J. STOKER
C. E. BURGESS
P. R. HALMOS
I. NIVEN
V. GANAPATHY IYER
T. G. OSTROM
G. SZEKERES
H. BUSEMANN
R. D. JAMES
M. M. SCHIFFER
F. WOLF
H. FEDERER

\section{SPONSORS}

\author{
UNIVERSITY OF BRITISH COLUMBIA \\ CALIFORNIA INSTITUTE OF TECHNOLOGY \\ UNIVERSITY OF CALIFORNIA \\ CALIFORNIA RESEARCH CORPORATION \\ MONTANA STATE UNIVERSITY \\ UNIVERSITY OF NEVADA \\ OREGON STATE COLLEGE \\ UNIVERSITY OF OREGON \\ UNIVERSITY OF SOUTHERN CALIFORNIA
}

\author{
STANFORD UNIVERSITY \\ UNIVERSITY OF UTAH \\ WASHINGTON STATE COLLEGE \\ UNIVERSITY OF WASHINGTON \\ AMERICAN MATHEMATICAL SOCIETY \\ HUGHES AIRCRAFT COMPANY
}

Mathematical papers intended for publication in the Pacific Journal of Mathematics should be typewritten (double spaced), and the author should keep a complete copy. Manuscripts may be sent to any of the editors. Manuscripts intended for the outgoing editors should be sent to their successors. All other communications to the editors should be addressed to the managing editor, Alfred Horn at the University of California, Los Angeles 24, California.

50 reprints of each article are furnished free of charge; additional copies may be obtained at cost in multiples of 50 .

The Pacific Journal of Mathematics is published quarterly, in March, June, September, and December. The price per volume (4 numbers) is $\$ 12.00$; single issues, $\$ 3.50$. Back numbers are available. Special price to individual faculty members of supporting institutions and to individual members of the American Mathematical Society: $\$ 4.00$ per volume; single issues, $\$ 1.25$.

Subscriptions, orders for back numbers, and changes of address should be sent to Pacific Journal of Mathematics, c/o University of California Press, Berkeley 4, California.

Printed at Kokusai Bunken Insatsusha (International Academic Printing Co., Ltd.), No. 10, 1-chome, Fujimi-cho, Chiyoda-ku, Tokyo, Japan.

* During the absence of E. G. Straus.

PUBLISHED BY PACIFIC JOURNAL OF MATHEMATICS, A NON-PROFIT CORPORATION COPYRIGHT 1956 BY PACIFIC JOURNAL OF MATHEMATICS 


\section{Pacific Journal of Mathematics}

\section{Vol. 6, No. $2 \quad$ December, 1956}

Louis Auslander, Remark on the use of forms in variational calculations .......................................... 209

Hubert Spence Butts, Jr. and Henry B. Mann, Corresponding residue systems in algebraic number fields ........................ 211

L. Carlitz and John Herbert Hodges, Distribution of matrices in a finite field............................................

Paul Civin and Bertram Yood, Invariant functionals ............... 231

David James Dickinson, Henry Pollak and G. H. Wannier, On a class of polynomials orthogonal over a denumerable set .................

Bernard Friedman and Luna Mishoe, Eigenfunction expansions associated with a non-self-adjoint differential equation ....................

Luna Mishoe and G. C. Ford, On the uniform convergence of a certain eigenfunction series .............................. 271

John W. Green, Mean values of harmonic functions on homothetic curves...........................................

Charles John August Halberg, Jr. and Angus E. Taylor, On the spectra of linked operators .....................................

Chuan Chih Hsiung, Some integral formulas for closed hypersurfaces in Riemannian space ................................... 291

Norman D. Lane, Differentiable points of arcs in conformal n-space 301

Louis F. McAuley, A relation between perfect separability, completeness, and normality in semi-metric spaces

G. Power and D. L. Scott-Hutton, The slow shearing motion of a liquid past a semi-infinite plane .............................

A. C. Schaeffer, Entire functions

Edward Silverman, An intrinsic inequality for Lebesgue area...

Choy-Tak Taam, Asymptotic relations between systems of differential equations.

Ti Yen, Quotient algebra of a finite $A W^{*}$-algebra ... 